\title{
The SGLT2 Inhibitor Dapagliflozin Significantly Improves the Peripheral Microvascular Endothelial Function in Patients with Uncontrolled Type 2 Diabetes Mellitus
}

\author{
Seigo Sugiyama ${ }^{1,2}$, Hideaki Jinnouchi ${ }^{1-3}$, Noboru Kurinami ${ }^{1}$, Kunio Hieshima ${ }^{1}$, \\ Akira Yoshida ${ }^{1}$, Katsunori Jinnouchi ${ }^{1}$, Hiroyuki Nishimura ${ }^{1}$, Tomoko Suzuki ${ }^{1}$, \\ Fumio Miyamoto $^{1}$, Keizo Kajiwara ${ }^{1,2}$ and Tomio Jinnouchi ${ }^{1,2}$
}

\begin{abstract}
:
Objective Sodium-glucose co-transporter 2 (SGLT2) inhibitors reduce cardiovascular events and decrease the body fat mass in patients with type 2 diabetes mellitus (T2DM). We examined whether or not the SGLT2-inhibitor dapagliflozin can improve the endothelial function associated with a reduction in abdominal fat mass.

Methods We prospectively recruited patients with uncontrolled [hemoglobin A1c (HbA1c) $>7.0 \%$ ] T2DM who were not being treated by SGLT2 inhibitors. Patients were treated with add-on dapagliflozin $(5 \mathrm{mg} /$ day $)$ or non-SGLT2 inhibitor medicines for 6 months to improve their HbA1c. We measured the peripheral microvascular endothelial function as assessed by reactive hyperemia peripheral arterial tonometry (RH-PAT) and calculated the natural logarithmic transformed value of the RH-PAT index (LnRHI). We then investigated changes in the LnRHI and abdominal fat area using computed tomography (CT).

Results The subjects were 54 patients with uncontrolled T2DM (72.2\% men) with a mean HbA1c of $8.1 \%$. The HbAlc was significantly decreased in both groups, with no significant difference between the groups. Dapagliflozin treatment, but not non-SGLT2 inhibitor treatment, significantly increased the LnRHI. The changes in the LnRHI were significantly greater in the dapagliflozin group than in the non-SGLT2 inhibitor group. Dapagliflozin treatment, but not non-SGLT2 inhibitor treatment, significantly decreased the abdominal visceral fat area, subcutaneous fat area (SFA), and total fat area (TFA) as assessed by CT and significantly increased the plasma adiponectin levels. The percentage changes in the LnRHI were significantly correlated with changes in the SFA, TFA, systolic blood pressure, and adiponectin.

Conclusion Add-on treatment with dapagliflozin significantly improves the glycemic control and endothelial function associated with a reduction in the abdominal fat mass in patients with uncontrolled T2DM.
\end{abstract}

Key words: sodium-glucose co-transporter 2 inhibitor, endothelial function, fat mass, type 2 diabetes

(Intern Med 57: 2147-2156, 2018)

(DOI: 10.2169/internalmedicine.0701-17)

\section{Introduction}

Sodium-glucose co-transporter 2 (SGLT2) inhibitors exert their glucose-lowering effects via the inhibition of SGLT2 protein in the kidney proximal tubule. This results in the im- provement of the glycemic control in patients with type 2 diabetes mellitus (T2DM) by an insulin-independent glucose-lowering action accompanied by body weight loss, mainly with a reduction in the fat mass (1-3). Obesity and fat accumulation are major fundamental pathogenic conditions in patients with T2DM (4). In patients with uncon-

${ }^{1}$ Diabetes Care Center, Jinnouchi Hospital, Japan, ${ }^{2}$ Cardiovascular Division, Diabetes Care Center, Jinnouchi Hospital, Japan and ${ }^{3}$ Division of Preventive Cardiology, Department of Cardiovascular Medicine, Kumamoto University Hospital, Japan

Received: December 21, 2017; Accepted: January 9, 2018; Advance Publication by J-STAGE: March 30, 2018

Correspondence to Dr. Seigo Sugiyama, seigosugiyama@jinnouchi.or.jp 
trolled T2DM under treatment with pre-existing anti-diabetic medications, physicians clinically expect greater improvement of the glycemic control through the novel glucoselowering benefits of add-on SGLT2 inhibitors $(5,6)$.

In cardiovascular safety studies of add-on treatment of SGLT2 inhibitors, the EMPA-REG OUTCOME ${ }^{\circledR}$ trial of empagliflozin and cardiovascular assessment study (CANVAS) of canagliflozin showed not only its cardiovascular safety but also its superiority with regard to reducing major adverse cardiovascular events in high-risk T2DM $(7,8)$. At present, the potential mechanisms of SGLT2 inhibitors for preventing cardiovascular event have not been fully determined (9). Recent clinical studies have shown that SGLT2 inhibitors have beneficial effects on the vascular function in patients with DM (10-12). However, the practical effects of add-on treatment of dapagliflozin on improving the glycemic control and microvascular endothelial dysfunction have not been investigated in patients with uncontrolled T2DM.

T2DM is considered a strong, major clinical risk factor for developing atherosclerosis and cardiovascular diseases (13). Vascular endothelial dysfunction is a significant and independent predictor of future cardiovascular events $(14,15)$. The presence of T2DM has been recognized as a crucial pathological condition leading to endothelial dysfunction in clinical practice (16). Increased attention has been focused on investigating and developing practical strategies for improving the vascular endothelial function clinically (17). Glucose-lowering therapies that may show favorable effects on the endothelial function are thought to have additionally clinical value for managing DM (18). SGLT2 inhibitors have been reported to improve glycemic control by reducing body fat mass $(2,3,19)$. Therefore, SGLT2 inhibitors could provide clinical and cardiovascular benefits by improving the endothelial function and decreasing the body fat mass.

In the present study, we hypothesized that add-on dapagliflozin treatment would improve the glycemic control and endothelial function by reducing the abdominal fat mass. Therefore, we investigated the effects of add-on dapagliflozin therapy on the peripheral microvascular endothelial function in patients with uncontrolled T2DM [hemoglobin A1c $(\mathrm{HbA} 1 \mathrm{c})>7.0 \%$ ]. The peripheral microvascular endothelial function was assessed by a reactive hyperemiaperipheral arterial tonometry (RH-PAT) examination and abdominal fat area was measured by computed tomography (CT).

\section{Materials and Methods}

\section{Study population and study protocol}

We prospectively recruited Japanese patients with stable but uncontrolled (HbA1c >7.0\%) T2DM without severe obesity [body mass index $(\mathrm{BMI})<35 \mathrm{~kg} / \mathrm{m}^{2}$ ] who had not taken SGLT2 inhibitors before from the Diabetes Care Center in Jinnouchi Hospital between 2014 and 2017. The exclusion criteria were as follows: type $1 \mathrm{DM}$, insulin-dependent condition [fasting C-peptide immunoreactivity (CPR) $<0.6 \mathrm{ng} /$ $\mathrm{mL}$, a history of ketoacidosis, age $>80$ years, unstable cardiovascular disease, active inflammation, severe liver disease, dementia, chronic kidney disease (estimated glomerular filtration rate $<30 \mathrm{~mL} / \mathrm{min} / 1.73 \mathrm{~m}^{2}$ ), urinary tract infection, cancer, and those who could not remain standing to have a body composition examination. Patients with newly diagnosed DM without any treatments were also excluded. We performed an RH-PAT examination in the morning, and patients with good endothelial function [reactive hyperemia index $($ RHI $)>2.10$ ] were also excluded.

The attending physicians at Jinnouchi Hospital nonrandomly separated the enrolled patients into two groups: the dapagliflozin group and the non-SGLT2 inhibitor group. Inclusion in the treatment arm was left to the physicians' discretion. Patients in the dapagliflozin group received their ongoing treatments and additional dapagliflozin $(5 \mathrm{mg} /$ day) for 6 months. Patients in the non-SGLT2 inhibitor group received their ongoing treatments along with intensification of glucose-lowering medications (increased doses and addition of other types of medications) without SGLT2 inhibitors to achieve an HbA1c level $<7.0 \%$ by the end of the study period.

Before starting treatment, a fasting blood sample was collected from the antecubital vein in the morning. The body composition was measured by a bioelectrical impedance analysis using the InBody $770^{\circledR}$ (Biospace, Seoul, Korea) and plain abdominal CT (Aquilion $\mathrm{CXL}^{\circledR}$; Toshiba, Tokyo, Japan). After 6 months of treatment, another fasting blood sample was collected, and a second body composition analysis using the InBody $770^{\circledR}$, plain abdominal CT, and an RHPAT examination was performed.

The primary outcome was the dapagliflozin-induced changes in the endothelial function as assessed by an RHPAT examination as a prospective, parallel-arm, open-label study. This study was conducted in accordance with the Declaration of Helsinki. The study protocol was approved by the Human Ethics Review Committee of Jinnouchi Hospital (2014-5-4), and a signed consent form was obtained from each patient. This study was registered under the UMIN protocol registration system (ID UMIN0000333354).

\section{Measurement of the peripheral microvascular endo- thelial function by an RH-PAT examination}

The principles and details of the RH-PAT examination have been described previously $(20,21)$. In brief, we volumetrically evaluated the peripheral microvascular endothelial function in the fingertip by RH-PAT using an Endo-PAT $2000^{\circledR}$ device (Itamar Medical, Caesarea, Israel). Measurements were taken when patients were in a stable condition and in the fasting state in the early morning before taking their medications. Patients were examined on a bed in the supine position after at least 10 minutes of rest in a temperature- and light-controlled environment. A blood pressure cuff was placed on the upper arm to be studied, while 
the contra-lateral arm served as a control. PAT probes were placed on one finger of each hand. After an equilibration period, the baseline pulse volume amplitude was measured for each fingertip for 5 minutes. The cuff was inflated to 60 $\mathrm{mmHg}$ above systolic pressure or $200 \mathrm{mmHg}$ for 5 minutes before being deflated to induce reactive hyperemia. After cuff deflation, PAT recordings were performed for $10 \mathrm{~min}$ utes, and RH-PAT data were automatically analyzed in real time using a software program (Endo-PAT2000 ${ }^{\circledR}$ software, version 3.5.4; Itamar Medical). The RHI reflects the extent of reactive hyperemia and was calculated as the ratio of the average amplitude of the PAT signal over 1.0 minutes starting 1.5 minutes after deflation of the cuff (control arm, A; occluded arm, C) divided by the average amplitude of the PAT signal at 2.5 minutes before inflation of the cuff (baseline: control arm, B; occluded arm, D) according to the following equation:

(C/D) / (A/B); (20)

We calculated the natural logarithmic transformation (Ln) of the RHI (LnRHI) (14).

Measurement of the body composition using a bioelectrical impedance analyzer (InBody770 $\left.{ }^{\circledR}\right)$

Anthropometric measurements were obtained in the standing position. Elementary body composition, including total fat mass and body fat percentage, was measured using a direct segmental multi-frequency bioelectrical impedance analyzer (InBody $770^{\circledR}$ ) (22). This analyzer processes 30 impedance measurements using 6 different frequencies $(1,5,50$, 250,500 , and $1,000 \mathrm{kHz}$ ) at each of 5 segments of the body (right arm, left arm, trunk, right leg, and left leg). In addition, measurements are processed by 15 reactance measurements using tetrapolar 8-point tactile electrodes at 3 different frequencies $(5,50$, and $250 \mathrm{kHz})$ at each of 5 segments of the body (right arm, left arm, trunk, right leg, and left leg) (23).

\section{Measurement of the visceral fat area and subcutane- ous fat area using abdominal CT}

On CT slices that were obtained at the umbilical level, the adipose tissue area located in the peritoneal cavity was defined as the visceral fat area (VFA: $\mathrm{cm}^{2}$ ), and the adipose tissue area underlying the skin layer was defined as the subcutaneous fat area $\left(\right.$ SFA: $\left.\mathrm{cm}^{2}\right)$. The VFA and SFA were measured on CT cross-sectional scans obtained at the umbilical level in the supine position (24) using a commercial software program based on the Japanese guidelines of obesity treatment (Japan Society for the Study of Obesity, in Japanese). The total fat area (TFA: $\mathrm{cm}^{2}$ ) was the sum of the SFA and VFA.

\section{Blood sampling and the measurement of clinical pa- rameters}

Fasting blood samples were collected from the antecubital vein in the morning. Blood analyses were conducted in the hospital laboratory to measure the levels of hemoglobin, he- matocrit, blood glucose, HbA1c, cholesterol, triglycerides, CPR, creatinine, and B-type natriuretic peptide (BNP). The levels of adiponectin, non-esterified fatty acid (NEFA), glucagon, and total ketone bodies were measured by SRL (Tokyo, Japan).

\section{Outcomes}

The primary endpoint was the treatment-induced changes in the endothelial function as assessed by an RH-PAT examination using an Endo-PAT2000 ${ }^{\circledR}$. We tested the superiority of the dapagliflozin-induced changes in the LnRHI compared with non-SGLT2 inhibitor therapy following a sixmonth course of treatment. The secondary outcome was the relationship between the percentage changes in the LnRHI and changes in fat mass and other clinical parameters.

\section{Statistical analyses}

Based on our preliminary examination at our hospital, a power analysis indicated that an enrollment of more than 48 patients was required to detect a mean difference in the change in the LnRHI of 0.15 in the dapagliflozin group and 0.05 in the non-SGLT2 inhibitor group, with a standard deviation of 0.15 , a power of $90 \%$, and a 2 -sided alpha of 0.05 . The results of normally distributed continuous variables (determined by the Shapiro-Wilk test) were expressed as the mean [standard deviation (SD)], while those of continuous variables with a skewed distribution were expressed as median values (interquartile range). Differences in the baseline characteristics of the two groups were analyzed by Student's $t$-test, the Mann-Whitney U test, or Fisher's exact test for categorical data, as appropriate. Either a paired Student's $t$-test or Wilcoxon's test was used to analyze the effect of each treatment. The difference between treatment groups in changes in the LnRHI from baseline to six months was also assessed using an analysis of covariance (ANCOVA) with adjustment for baseline measures as covariates (pre-LnRHI, age, duration of DM, pre-HbA1c, body weight, and systolic blood pressure).

To determine the relationships between changes in various clinical parameters and percentage changes in the LnRHI, correlations between variables of interest were analyzed using Pearson's correlation coefficient. Patients with an increase in the LnRHI $>15.0 \%$ were defined as having an improved endothelial function in the present study (25). A logistic regression analysis was used to evaluate the association between improvement in the endothelial function and baseline clinical variables, including the age, sex, BMI, HbAlc, fasting plasma glucose (FPG), lipid parameters, medications, and therapy allocation (dapagliflozin or nonSGLT2 inhibitor therapy). Associations between groups and all other parameters were analyzed first by a univariate logistic regression analysis. This was followed by a multivariate logistic regression analysis using the forced inclusion model, and the Hosmer-Lemeshow goodness-of-fit statistic was calculated. A p value $<0.05$ was considered statistically significant. Statistical analyses were performed using the 
Table 1. Baseline Clinical Characteristics.

\begin{tabular}{|c|c|c|c|c|}
\hline & Total patients $(n=54)$ & Dapagliflozin $(n=27)$ & Non-SGLT2i $(n=27)$ & $\mathrm{p}$ value \\
\hline Age (years) & $55.6 \pm 8.3$ & $55.2 \pm 8.7$ & $56.0 \pm 8.0$ & 0.75 \\
\hline Sex (male: \%) & $72.2 \%$ & $70.4 \%$ & $74.1 \%$ & 1.00 \\
\hline Body mass index $\left(\mathrm{kg} / \mathrm{m}^{2}\right)$ & $27.1 \pm 2.7$ & $27.6 \pm 2.2$ & $26.5 \pm 3.1$ & 0.13 \\
\hline Hypertension & $66.7 \%$ & $70.4 \%$ & $63.0 \%$ & 0.77 \\
\hline Dyslipidemia & $74.1 \%$ & $70.4 \%$ & $77.8 \%$ & 0.76 \\
\hline Current smoking & $24.1 \%$ & $22.2 \%$ & $25.9 \%$ & 1.00 \\
\hline Duration of diabetes (years) & $10.0(7.0-17.0)$ & $10.0(6.0-15.0)$ & $11.0(8.0-18.0)$ & 0.46 \\
\hline Hemoglobin A1c (\%) & $7.9(7.3-8.7)$ & $7.9(7.3-8.9)$ & $8.0(7.4-8.6)$ & 0.97 \\
\hline Fasting plasma glucose (mg/dL) & $143.2 \pm 38.7$ & $137.6 \pm 39.1$ & $148.8 \pm 38.2$ & 0.29 \\
\hline Anti-diabetic medicines (\%) & - & - & - & - \\
\hline Sulfonylureas (\%) & $25.9 \%$ & $33.3 \%$ & $18.5 \%$ & 0.35 \\
\hline Glinide $(\%)$ & $13.0 \%$ & $11.1 \%$ & $14.8 \%$ & 1.00 \\
\hline Metformin $(\%)$ & $94.4 \%$ & $96.3 \%$ & $92.4 \%$ & 1.00 \\
\hline Alpha-glucosidase inhibitor $(\%)$ & $13.0 \%$ & $7.4 \%$ & $18.5 \%$ & 0.42 \\
\hline Thiazolidinedione $(\%)$ & $11.1 \%$ & $14.0 \%$ & $7.4 \%$ & 0.67 \\
\hline DPP-4 inhibitor (\%) & $44.4 \%$ & $40.7 \%$ & $48.1 \%$ & 0.79 \\
\hline GLP-1 receptor agonist $(\%)$ & $11.1 \%$ & $14.8 \%$ & $7.4 \%$ & 0.67 \\
\hline Insulin $(\%)$ & $44.4 \%$ & $37.0 \%$ & $51.9 \%$ & 0.41 \\
\hline
\end{tabular}

SGLT2i: sodium glucose co-transporter 2 inhibitor, DPP-4: dipeptidyl peptidase, GLP-1: glucagon like peptide-1

Statistical Package for Social Sciences software program, version 23 (SPSS, IBM, Tokyo, Japan).

\section{Results}

\section{Baseline clinical characteristics of the study sub- jects}

A total of 54 Japanese patients with uncontrolled T2DM were enrolled and non-randomly assigned to the 2 groups. All of the patients completed the study protocol. The clinical baseline characteristics of the total patients and those of each group are shown in Table 1 . The mean age was 56.1 years, $72.2 \%$ were men, the mean BMI was $27.1 \mathrm{~kg} / \mathrm{m}^{2}$, the mean HbA1c was $8.1 \%$, and the mean FPG level was 143.2 $\mathrm{mg} / \mathrm{dL}$. At enrollment, $44.4 \%$ of patients were treated with insulin, and the frequency of the baseline anti-diabetic medications was not significantly different between the groups. The baseline characteristics of patients in the dapagliflozin group were similar to those of the non-SGLT2 inhibitor group. The BMI, HbA1c levels, FPG levels, and duration of DM were not significantly different between the groups.

\section{Changes in the HbA1c, FPG, glucagon, and CPR}

At the end of the study period, $5 \mathrm{mg} /$ day of dapagliflozin was used for the dapagliflozin group. In the non-SGLT2 inhibitor group, the doses of previously prescribed medications were increased in $82.6 \%$ of the patients, and new medications were administered to $33.3 \%$ of the patients. The total patients in both treatment groups showed a significant improvement in HbA1c [median (interquartile range): pretreatment to post-treatment, $7.9 \%(7.3-8.7 \%)$ to $6.9 \%(6.5-$ $7.5 \%), \mathrm{p}<0.01]$ and FPG levels (mean \pm SD: $143.2 \pm 38.7$ to
$125.1 \pm 27.2 \mathrm{mg} / \mathrm{dL}, \mathrm{p}<0.01)$ at 6 months. As shown in Table 2, the HbA1c levels were significantly decreased in both groups, while the absolute changes in the HbAlc and FPG levels were not significantly different between the dapagliflozin and non-SGLT2 inhibitor groups. We did not find any marked changes in the fasting plasma glucagon levels in either group. Dapagliflozin treatment significantly decreased the fasting blood CPR levels.

\section{Changes in the body weight, total body fat mass, and abdominal fat area as assessed by CT}

Dapagliflozin treatment, but not non-SGLT2 inhibitor treatment, for 6 months significantly decreased the body weight $($ mean $\pm \mathrm{SD} ;-4.4 \pm 3.2 \%, \mathrm{p}<0.01)$ and total fat mass $($ mean $\pm S D ;-12.1 \pm 11.3 \%, \mathrm{p}<0.01)$ compared with the baseline (Table 2). Dapagliflozin treatment, but not non-SGLT2 inhibitor treatment, also significantly decreased the waist circumference (mean $\pm \mathrm{SD} ;-3.2 \pm 5.4 \%, \mathrm{p}<0.01$ ), abdominal VFA $($ mean \pm SD; $-21.2 \pm 15.2 \%, \mathrm{p}<0.01)$, SFA (mean \pm SD; $-12.4 \pm$ $15.1 \%, \mathrm{p}<0.01)$, and TFA (mean $\pm \mathrm{SD} ;-14.9 \pm 13.3 \%, \mathrm{p}<0.01)$ compared with the baseline.

\section{Changes in the blood pressure, heart rate, and other metabolic parameters}

As shown in Table 3, dapagliflozin treatment, but not non-SGLT2 inhibitor treatment, significantly decreased the blood pressure at six months compared with the baseline, as reported previously (26). Furthermore, the heart rate in the dapagliflozin treatment group was also significantly decreased at six months compared with the baseline. The hematocrit and hemoglobin levels were significantly increased in the dapagliflozin treatment group at six months compared with the baseline, with no marked changes in the non- 
Table 2. Changes in Body Weight, Body Composition, and Glucose Metabolic Parameters.

\begin{tabular}{|c|c|c|c|c|c|c|}
\hline & \multicolumn{2}{|c|}{ Dapagliflozin $(\mathrm{n}=27)$} & \multirow{2}{*}{$\mathrm{p}$ value } & \multicolumn{2}{|c|}{ Non-SGLT2i (n=27) } & \multirow{2}{*}{$\mathrm{p}$ value } \\
\hline & Baseline & 6 months & & Baseline & 6 months & \\
\hline HbA1c (\%) & $7.9(7.3-8.9)$ & $6.7(6.3-7.4)$ & $<0.01$ & $8.0(7.4-8.6)$ & $7.0(6.7-8.0)$ & $<0.01$ \\
\hline Absolute change (\%) & \multicolumn{2}{|c|}{$-1.2(-1.6--0.5)$} & \multicolumn{4}{|c|}{$-0.7(-1.1--0.3)$} \\
\hline Fasting plasma glucose $(\mathrm{mg} / \mathrm{dL})$ & $137.6 \pm 39.1$ & $116.4 \pm 23.1$ & $<0.01$ & $148.8 \pm 38.2$ & $133.9 \pm 28.5$ & 0.07 \\
\hline Absolute change (mg/dL) & \multicolumn{2}{|c|}{$-21.1 \pm 26.4$} & \multicolumn{4}{|c|}{$-14.9 \pm 41.2$} \\
\hline Glucagon (pg/mL) & $145.8 \pm 23.7$ & $145.2 \pm 22.1$ & 0.92 & $143.2 \pm 28.2$ & $139.3 \pm 28.4$ & 0.59 \\
\hline Absolute change (pg/mL) & \multicolumn{2}{|c|}{$-0.57 \pm 28.1$} & \multicolumn{4}{|c|}{$-3.85 \pm 35.9$} \\
\hline CPR (ng/mL) & $2.08 \pm 0.75$ & $1.67 \pm 0.72$ & $<0.01$ & $1.56 \pm 0.93$ & $1.55 \pm 1.01$ & 0.87 \\
\hline Absolute change (ng/mL) & \multicolumn{2}{|c|}{$-0.41(-0.69--0.09)$} & $\dagger$ & \multicolumn{2}{|c|}{$-0.10(-0.32-0.12)$} & \\
\hline Body mass index $\left(\mathrm{kg} / \mathrm{m}^{2}\right)$ & $27.6 \pm 2.2$ & $26.4 \pm 2.4$ & $<0.01$ & $26.5 \pm 3.1$ & $26.1 \pm 3.1$ & 0.13 \\
\hline Absolute change $\left(\mathrm{kg} / \mathrm{m}^{2}\right)$ & \multicolumn{2}{|c|}{$-1.2 \pm 0.9$} & $\dagger$ & \multicolumn{2}{|c|}{$-0.3 \pm 1.0$} & \\
\hline Body weight $(\mathrm{kg})$ & $75.3 \pm 7.4$ & $72.0 \pm 7.1$ & $<0.01$ & $72.2 \pm 8.9$ & $71.4 \pm 9.2$ & 0.13 \\
\hline Absolute change (kg) & \multicolumn{2}{|c|}{$-3.3 \pm 2.6$} & $\dagger$ & \multicolumn{2}{|c|}{$-0.8 \pm 2.8$} & \\
\hline Waist circumference $(\mathrm{cm})$ & $96.3 \pm 6.4$ & $93.4 \pm 6.9$ & $<0.01$ & $92.8 \pm 7.3$ & $91.6 \pm 7.7$ & 0.20 \\
\hline Absolute change (cm) & \multicolumn{2}{|c|}{$-3.2 \pm 5.4$} & \multicolumn{4}{|c|}{$-1.2 \pm 4.7$} \\
\hline Total fat mass $(\mathrm{kg})$ & $25.1 \pm 5.3$ & $22.2 \pm 5.8$ & $<0.01$ & $21.2 \pm 6.5$ & $20.8 \pm 6.8$ & 0.43 \\
\hline Absolute change (kg) & \multicolumn{2}{|c|}{$-2.9 \pm 2.8$} & $\dagger$ & \multicolumn{2}{|c|}{$-0.4 \pm 2.7$} & \\
\hline Body fat percentage (\%) & $33.3 \pm 6.6$ & $30.8 \pm 7.6$ & $<0.01$ & $29.2 \pm 8.0$ & $28.8 \pm 8.2$ & 0.55 \\
\hline Absolute change (\%) & \multicolumn{2}{|c|}{$-2.5 \pm 2.9$} & $\dagger$ & \multicolumn{2}{|c|}{$-0.3 \pm 2.8$} & \\
\hline Abdominal VFA $\left(\mathrm{cm}^{2}\right)$ & $129.0 \pm 48.6$ & $102.3 \pm 44.5$ & $<0.01$ & $119.2 \pm 61.0$ & $115.6 \pm 63.2$ & 0.45 \\
\hline Absolute change $\left(\mathrm{cm}^{2}\right)$ & \multicolumn{2}{|c|}{$-18.9(-39.5--10.7)$} & $\dagger$ & \multicolumn{2}{|c|}{$-4.6(-15.9-20.5)$} & \\
\hline Abdominal SFA $\left(\mathrm{cm}^{2}\right)$ & $\begin{array}{c}200.9 \\
(144.4-266.6)\end{array}$ & $\begin{array}{c}166.6 \\
(129.7-253.5)\end{array}$ & $<0.01$ & $\begin{array}{c}163.8 \\
(128.1-187.7)\end{array}$ & $\begin{array}{c}156.8 \\
(130.8-204.4)\end{array}$ & 0.63 \\
\hline Absolute change $\left(\mathrm{cm}^{2}\right)$ & \multicolumn{2}{|c|}{$-18.3(-35.1--7.6)$} & $\dagger$ & \multicolumn{2}{|c|}{$-2.5(-11.3-11.3)$} & \\
\hline Abdominal TFA $\left(\mathrm{cm}^{2}\right)$ & $340.2 \pm 90.7$ & $287.2 \pm 90.7$ & $<0.01$ & $283.7 \pm 87.9$ & $280.3 \pm 97.4$ & 0.68 \\
\hline Absolute change $\left(\mathrm{cm}^{2}\right)$ & \multicolumn{2}{|c|}{$-35.4(-59.8--23.6)$} & $\dagger$ & \multicolumn{2}{|c|}{$-7.1(-25.1--22.5)$} & \\
\hline
\end{tabular}

$\dagger \mathrm{p}<0.01, \neq \mathrm{p}<0.05$ Dapagliflozin versus Non-SGLT2i. SGLT2i: sodium glucose co-transporter 2 inhibitor, HbA1c: hemoglobin A1c, VFA: visceral fat area, SFA: subcutaneous fat area, TFA: total fat area, NEFA: non esterified free fatty acid, CPR: C-peptide immunoreactivity

SGLT2 inhibitor group. The BNP levels appeared to be decreased at six months compared with the baseline in the dapagliflozin treatment group, but this was not significant. The absolute changes in the BNP levels were not significantly different between the two groups. The total cholesterol levels, low-density lipoprotein cholesterol levels, NEFA levels, and estimated glomerular filtration rate did not show significant changes in either group. The plasma total ketone body levels were significantly increased in the dapagliflozin group at six months compared with the baseline, but there were no marked changes in the non-SGLT2 inhibitor group (Table 3). The triglyceride levels were significantly decreased in the dapagliflozin group at six months compared with the baseline, but the absolute changes in triglyceride levels were not significantly different between the two groups. Dapagliflozin treatment, but not non-SGLT2 inhibitor treatment, significantly increased the plasma adiponectin levels at six months compared with the baseline. The absolute changes in adiponectin levels were significantly greater in the dapagliflozin group than in the non-SGLT2 inhibitor group.

\section{Changes in the endothelial function as assessed by an RH-PAT examination}

During 6 months of treatment, the peripheral microvascu- lar endothelial function, as assessed by the LnRHI, was significantly improved after glucose-lowering therapy in the total patients (LnRHI: $0.450 \pm 0.125$ to $0.592 \pm 0.228$, p<0.01). Add-on dapagliflozin-therapy, but not non-SGLT2 inhibitor therapy, resulted in a significant intra-group improvement in the endothelial function (dapagliflozin group: $0.450 \pm 0.135$ to $0.662 \pm 0.230, \mathrm{p}<0.01$; non-SGLT2-inhibitor group: $0.450 \pm$ 0.118 to $0.522 \pm 0.208, \mathrm{p}=0.09$; Table 3 ). With regard to inter-group comparisons, the absolute changes and percentage changes in the LnRHI were significantly greater in the dapagliflozin group than in the non-SGLT2 inhibitor group ( $p=0.028$; Table 3 and Fig. 1). An ANCOVA with adjustment for the various baseline parameters revealed that the changes in the LnRHI were significantly greater in the dapagliflozin group than in the non-SGLT2 inhibitor group (ANCOVA, p=0.034).

\section{A logistic regression analysis for improvements in the endothelial function}

The proportion of patients with an improved endothelial function (percentage increase in LnRHI $>15 \%$ ) was $74.1 \%$ in the dapagliflozin group and $40.7 \%$ in the non-SGLT2 inhibitor group (Fig. 2, p=0.01). A univariate logistic regression analysis showed that dapagliflozin therapy was signifi- 
Table 3. Changes in Blood Pressure, Heart Rate, and Laboratory Parameters.

\begin{tabular}{|c|c|c|c|c|c|c|}
\hline & \multicolumn{2}{|c|}{ Dapagliflozin $(\mathrm{n}=27)$} & \multirow{2}{*}{$\mathrm{p}$ value } & \multicolumn{2}{|c|}{ Non-SGLT2i $(\mathrm{n}=27)$} & \multirow{2}{*}{$\mathrm{p}$ value } \\
\hline & Baseline & 6 months & & Baseline & 6 months & \\
\hline Systolic BP (mmHg) & $130.3 \pm 15.3$ & $121.9 \pm 13.1$ & $<0.01$ & $131.0 \pm 13.4$ & $129.1 \pm 16.0$ & 0.52 \\
\hline Absolute change $(\mathrm{mmHg})$ & \multicolumn{2}{|c|}{$-6.0(-11.0--1.0)$} & \multicolumn{4}{|c|}{$-3.0(-9.0-8.0)$} \\
\hline Diastolic BP (mmHg) & $78.0 \pm 8.3$ & $73.2 \pm 7.0$ & 0.015 & $76.3 \pm 7.5$ & $75.5 \pm 10.3$ & 0.57 \\
\hline Absolute change (mmHg) & \multicolumn{2}{|c|}{$-4.8 \pm 9.6$} & \multicolumn{4}{|c|}{$-0.8 \pm 7.3$} \\
\hline Heart rate (beat/min) & $78.3 \pm 6.6$ & $73.5 \pm 8.0$ & $<0.01$ & $76.1 \pm 11.3$ & $76.6 \pm 9.9$ & 0.71 \\
\hline Absolute change (beat/min) & \multicolumn{2}{|c|}{$-4.8 \pm 7.4$} & $\dagger$ & \multicolumn{2}{|c|}{$0.5 \pm 7.1$} & \\
\hline Hematocrit (\%) & $43.1 \pm 3.8$ & $45.8 \pm 3.8$ & $<0.01$ & $42.4 \pm 4.5$ & $42.3 \pm 3.4$ & 0.92 \\
\hline Absolute change (\%) & \multicolumn{2}{|c|}{$2.1(1.2-3.8)$} & $\dagger$ & \multicolumn{2}{|c|}{$0.1(-3.1-1.6)$} & \\
\hline Hemoglobin (g/dL) & $14.6 \pm 1.3$ & $15.4 \pm 1.4$ & $<0.01$ & $14.3 \pm 1.9$ & $14.2 \pm 1.3$ & 0.91 \\
\hline Absolute change (g/dL) & \multicolumn{2}{|c|}{$0.5(0-1.4)$} & $\dagger$ & \multicolumn{2}{|c|}{$0.2(-0.5-0.4)$} & \\
\hline $\mathrm{BNP}(\mathrm{pg} / \mathrm{mL})$ & $9.5(4.4-20.6)$ & $8.2(5.0-13.0)$ & 0.07 & $9.9(4.7-17.9)$ & $8.8(4.5-14.6)$ & 0.54 \\
\hline Absolute change (pg/mL) & \multicolumn{2}{|c|}{$-0.7(-9.5-1.0)$} & \multicolumn{4}{|c|}{$-0.4(-4.3-2.6)$} \\
\hline eGFR $\left(\mathrm{mL} / \mathrm{min} / 1.73 \mathrm{~m}^{2}\right)$ & $75.4 \pm 15.4$ & $74.6 \pm 15.2$ & 0.54 & $73.2 \pm 11.8$ & $73.6 \pm 13.0$ & 0.81 \\
\hline Absolute change & \multicolumn{2}{|c|}{$-0.9 \pm 7.2$} & \multicolumn{4}{|c|}{$0.4 \pm 7.3$} \\
\hline Total cholesterol (mg/dL) & $159.4 \pm 37.9$ & $157.7 \pm 31.9$ & 0.71 & $157.9 \pm 29.2$ & $154.1 \pm 34.4$ & 0.44 \\
\hline Absolute change (mg/dL) & \multicolumn{2}{|c|}{$-1.7 \pm 23.2$} & \multicolumn{4}{|c|}{$-3.8 \pm 25.3$} \\
\hline HDL-cholesterol (mg/dL) & $46.5 \pm 10.7$ & $51.3 \pm 12.3$ & $<0.01$ & $44.1 \pm 8.9$ & $45.8 \pm 10.3$ & 0.20 \\
\hline Absolute change (mg/dL) & \multicolumn{2}{|c|}{$4.0(0.0-7.0)$} & $\ddagger$ & \multicolumn{2}{|c|}{$0.0(-3.0-5.0)$} & \\
\hline LDL-cholesterol (mg/dL) & $87.9 \pm 30.7$ & $87.0 \pm 25.7$ & 0.79 & $89.0 \pm 24.1$ & $85.5 \pm 29.0$ & 0.40 \\
\hline Absolute change $(\mathrm{mg} / \mathrm{dL})$ & \multicolumn{2}{|c|}{$-0.9 \pm 17.8$} & \multicolumn{4}{|c|}{$-3.6 \pm 21.6$} \\
\hline Triglyceride (mg/dL) & $109(68-145)$ & $86(65-106)$ & $<0.01$ & $107(83-172)$ & $100(78-156)$ & 0.05 \\
\hline Absolute change (mg/dL) & \multicolumn{2}{|c|}{$-19(-52-4)$} & \multicolumn{4}{|c|}{$-6(-26-3)$} \\
\hline $\operatorname{NEFA}(\mu \mathrm{Eq} / \mathrm{L})$ & $\begin{array}{c}431.5 \\
(281.8-593.0)\end{array}$ & $\begin{array}{c}394.0 \\
(275.5-577.5)\end{array}$ & 0.95 & $\begin{array}{c}385.0 \\
(323.0-500.0)\end{array}$ & $\begin{array}{c}394.0 \\
(302.3-494.5)\end{array}$ & 0.80 \\
\hline Absolute change (mEq/L) & \multicolumn{2}{|c|}{$22.5(-168.8-144.0)$} & \multicolumn{4}{|c|}{$17.0(-71-139.3)$} \\
\hline Total ketone body $(\mu \mathrm{mol} / \mathrm{L})$ & $\begin{array}{c}73.0 \\
(57.0-119.0)\end{array}$ & $\begin{array}{c}156.0 \\
(80.0-276.0)\end{array}$ & $<0.01$ & $\begin{array}{c}69.0 \\
(49.5-124.0)\end{array}$ & $\begin{array}{c}81.0 \\
(53.0-131.3)\end{array}$ & 0.56 \\
\hline Absolute change $(\mu \mathrm{mol} / \mathrm{L})$ & \multicolumn{2}{|c|}{$63.0(2.0-176.0)$} & $\dagger$ & \multicolumn{2}{|c|}{$7.5(-43.3-44.3)$} & \\
\hline Adiponectin $(\mu \mathrm{g} / \mathrm{mL})$ & $5.8(4.5-7.4)$ & $7.2(6.0-9.8)$ & $<0.01$ & $5.8(5.7-8.0)$ & $6.5(5.1-8.0)$ & 0.06 \\
\hline Absolute change $(\mu \mathrm{g} / \mathrm{mL})$ & $1.0(C$ & - 1.7) & $\ddagger$ & 0.5 & $-0.9)$ & \\
\hline LnRHI & $0.450 \pm 0.135$ & $0.662 \pm 0.230$ & $<0.01$ & $0.450 \pm 0.118$ & $0.522 \pm 0.208$ & 0.09 \\
\hline Absolute change & 0.21 & .252 & $\ddagger$ & 0.07 & .213 & \\
\hline
\end{tabular}

$\dagger \mathrm{p}<0.01, \hbar \mathrm{p}<0.05$ Dapagliflozin versus Non-SGLT2i. SGLT2i: sodium glucose co-transporter 2 inhibitor, BP: blood pressure, BNP: B-type natriuretic peptide, eGFR: estimated glomerular filtration rate, NEFA: non-esterified fatty acid, LnRHI: natural logarithmic transformation of reactive-hyperemia index

cantly correlated with an improvement in the endothelial function (Table 4). A forced inclusion multivariate logistic regression analysis with the BMI and dapagliflozin therapy showed that only dapagliflozin therapy was significantly correlated with an improvement in the endothelial function (odds ratio: 3.931, 95\% confidence interval: 1.217 to 12.695, $\mathrm{p}=0.022)$. The Hosmer-Lemeshow statistic was appropriate $(\mathrm{p}=0.14)$.

\section{The association between changes in the clinical and metabolic parameters and changes in the LnRHI}

To determine the factors associated with improvement in the endothelial function as assessed by the percentage increase in the LnRHI and the changes in various clinical and laboratory parameters during the treatment period, we investigated the correlation coefficients between the percentage increase in the LnRHI and changes in the variables. The percentage change in the LnRHI was not significantly corre- lated with changes in the body weight, BMI, HbAlc levels, or FBG levels (Table 5). However, the percentage change in the LnRHI was significantly correlated with changes in the TFA, SFA, systolic blood pressure, and adiponectin levels. Changes in the waist circumference, as a simple anthropometric parameter of abdominal fat deposition, showed a non-significant correlation with the percentage change in the LnRHI.

\section{Discussion}

In patients with uncontrolled T2DM, add-on treatment of the SGLT2 inhibitor dapagliflozin for 6 months significantly improved the peripheral microvascular endothelial function as assessed by an RH-PAT examination. Dapagliflozin is a clinically useful and promising anti-diabetic treatment tool for improving the glycemic control and endothelial function, accompanied by a reduced blood pressure and abdominal fat 


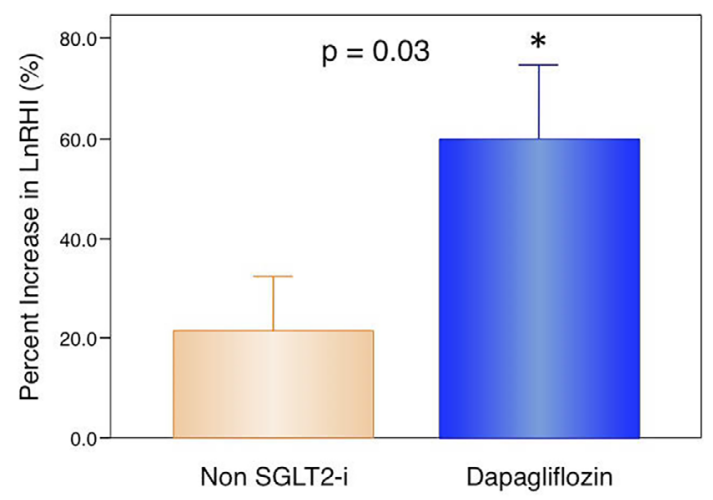

Figure 1. Percentage changes in the peripheral microvasular endothelial function as assessed by an RH-PAT examination in dapagliflozin therapy and non-SGLT2 inhibitor therapy. Bar graphs depict the changes in the natural logarithmic transformation of reactive hyperemia index (LnRHI) values (mean and standard error) in patients with dapagliflozin therapy $(n=27)$ or non-sodium-glucose co-transporter 2 (SGLT2) inhibitor therapy $(n=27)$. Percentage increase in LnRHI=[(LnRHI after 6 months of therapy)-(LnRHI at enrollment)] $\times 100 /$ (LnRHI at enrollment). With regard to the inter-group comparison, the changes in the LnRHI in patients with dapagliflozin therapy were significantly greater than those in the patients with nonSGLT2 inhibitor therapy (unpaired t-test, $p=0.03$ ). Six months of dapagliflozin therapy significantly increased the LnRHI compared with the baseline (paired t-test, ${ }^{*} \mathbf{p}<0.01$ ).

mass and increased plasma adiponectin levels in patients with T2DM.

$\mathrm{DM}$ is a major clinical risk factor for developing atherosclerosis and endothelial dysfunction, leading to an increased occurrence of cardiovascular complications (13). A meta-analysis showed that intensive glucose-lowering therapies were markedly effective at reducing the rate of myocardial infarction and coronary heart diseases (27). Therefore, effective glucose-lowering treatment strategies that have a favorable action on the endothelial function are thought to have additional clinical value in the management of DM (18). Thiazolidinediones significantly improved the endothelial function in a meta-analysis (28). In addition, Matsubara et al. showed that the DPP-4 inhibitor sitagliptin significantly improved the endothelial function in patients with T2DM and coronary artery disease (21). Torimoto et al. found that the glucagon-like peptide-1 receptor agonist exenatide improved postprandial vascular endothelial dysfunction after the meal loading test (29). In the present study, we clearly showed that add-on dapagliflozin for six months significantly improved the microvascular endothelial function in patients with uncontrolled T2DM. In cardiovascular safety studies, such as the EMPA-REG OUTCOME ${ }^{\circledR}$ trial and CANVAS program, add-on therapies of SGLT2 inhibitors proved effective in preventing major adverse cardiovascular events in high-risk T2DM patients $(7,8)$. SGLT2 inhibitors exert their novel glucose-lowering effects via insulin-

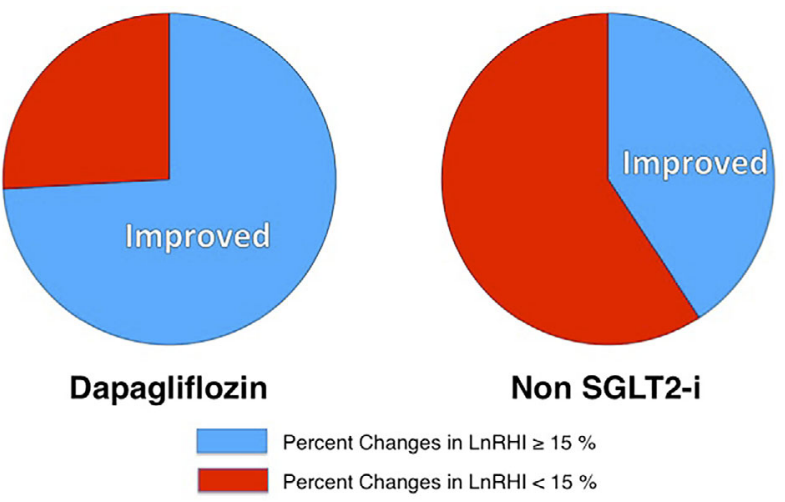

Figure 2. Pie charts of the proportion of patients with an improved endothelial function who received dapagliflozin therapy and non-SGLT2 inhibitor therapy. The proportion of patients with an improved endothelial function was $74.1 \%$ in the dapagliflozin group and $40.7 \%$ in the non-SGLT2 inhibitor group ( $p=0.013$ ). LnRHI: natural logarithmic transformation of the reactive hyperemia index, SGLT2-I: sodium-glucose cotransporter 2 inhibitor

independent mechanisms (5). In the present study, dapagliflozin exerted a beneficial vascular action by improving the microvascular endothelial function. Therefore, dapagliflozin may be a promising medication for improving the glycemic control and resolving endothelial dysfunction in patients with uncontrolled T2DM.

The detailed mechanisms underlying the prevention of cardiovascular events by SGLT2 inhibitors have not been fully determined. However, some potential vascular benefits of reducing the blood pressure and arterial stiffness have been reported (9). Shigiyama et al. recently demonstrated that add-on dapagliflozin with low-dose metformin tended to improve the endothelial function as assessed by flowmediated vasodilation (FMD) compared with an increasing dose of metformin in the early stage of T2DM (11). In their sub-group analysis, dapagliflozin significantly improved the endothelial function only in patients with uncontrolled T2 DM. They suggested that dapagliflozin might improve the endothelial function through a reduction in the oxidative stress as evaluated by urinary levels of 8-hydroxy-2'deoxyguanosin (11). Solini et al. recently reported that 2day dapagliflozin treatment rapidly and significantly improved the endothelial function as assessed by FMD in patients with T2DM (12). They also proposed a potential mechanism underlying the dapagliflozin-induced improvement of the vascular function via a reduction in the oxidative stress as assessed by urinary isoprostanes.

We cannot speculate on the possible involvement of reducing oxidative stress in improving the endothelial function by dapagliflozin in our study because we did not measure any markers of oxidative stress. Dapagliflozin therapy may reduce oxidative stress through several pathways and metabolic changes, including improvement of the glycemic control, elevation of adiponectin, and a reduction in triglyceride levels, the blood pressure, the body weight, and the fat 
Table 4. Logistic Regression Analysis for the Improvement of Microvascular Endothelial Function among Baseline Factors.

\begin{tabular}{|c|c|c|c|c|c|c|}
\hline \multirow{2}{*}{ Baseline variable } & \multicolumn{3}{|c|}{ Univariate logistic regression } & \multicolumn{3}{|c|}{$\begin{array}{l}\text { Multivariate logistic regression } \\
\text { using forced inclusion model }\end{array}$} \\
\hline & OR & $95 \% \mathrm{CI}$ & $\mathrm{p}$ & OR & $95 \% \mathrm{CI}$ & $\mathrm{p}$ \\
\hline Age (per year) & 1.056 & 0.986 to 1.132 & 0.120 & & - & \\
\hline Gender (male) & 1.159 & 0.345 to 3.893 & 0.811 & & - & \\
\hline Body mass index (per 1.0) & 1.107 & 0.902 to 1.359 & 0.329 & 1.056 & 0.851 to 1.311 & 0.620 \\
\hline Body weight (per 1.0; kg) & 1.014 & 0.949 to 1.083 & 0.687 & & - & \\
\hline Waist circumference (per $1.0 ; \mathrm{cm}$ ) & 1.033 & 0.955 to 1.117 & 0.419 & & - & \\
\hline Total fat mass $($ per $1.0 ; \mathrm{kg})$ & 1.067 & 0.973 to 1.170 & 0.167 & & - & \\
\hline Body fat percentage (per $1.0 ; \%$ ) & 1.048 & 0.972 to 1.130 & 0.218 & & - & \\
\hline Hypertension (yes) & 4.167 & 0.664 to 26.31 & 0.177 & & - & \\
\hline Dyslipidemia (yes) & 0.667 & 0.196 to 2.267 & 0.516 & & - & \\
\hline Current smoker (yes) & 1.210 & 0.345 to 4.245 & 0.776 & & - & \\
\hline Hemoglobin A1c (per 1.0; \%) & 1.320 & 0.744 to 2.342 & 0.343 & & - & \\
\hline FPG (per $1.0 ; \mathrm{mg} / \mathrm{dL})$ & 1.005 & 0.990 to 1.019 & 0.525 & & - & \\
\hline CPR (per 1.0; ng/mL) & 0.743 & 0.390 to 1.417 & 0.368 & & - & \\
\hline Duration (per 1.0; years) & 1.059 & 0.971 to 1.155 & 0.194 & & - & \\
\hline Abdominal VFA (per $1.0 ; \mathrm{cm}^{2}$ ) & 1.001 & 0.991 to 1.011 & 0.829 & & - & \\
\hline Abdominal SFA (per $1.0 ; \mathrm{cm}^{2}$ ) & 1.005 & 0.997 to 1.014 & 0.205 & & - & \\
\hline Dapagliflozin therapy (yes) & 4.156 & 1.312 to 13.169 & 0.015 & 3.931 & 1.217 to 12.695 & 0.022 \\
\hline
\end{tabular}

Hosmer-Lemeshow $\mathrm{p}=0.14$ in multivariate analysis.

OR: odds ratio, CI: confidence interval, FPG: fasting plasma glucose, CPR: C-peptide immunoreactivity, VFA: visceral fat area, SFA: subcutaneous fat area

Table 5. Correlation between Changes in Improvement of Endothelial Function as Assessed by the Percentage Increase in LnRHI and Clinical Variables.

\begin{tabular}{lrc}
\hline & $\mathrm{r}$ & $\mathrm{p}$ value \\
\hline Changes in body mass index $\left(\mathrm{kg} / \mathrm{m}^{2}\right)$ & -0.204 & 0.139 \\
Changes in body in body weight $(\mathrm{kg})$ & -0.189 & 0.170 \\
Changes in waist circumference $(\mathrm{cm})$ & -0.256 & 0.062 \\
Changes in total fat mass $(\mathrm{kg})$ & -0.223 & 0.106 \\
Changes in body fat percentage $(\%)$ & -0.218 & 0.114 \\
Changes in hemoglobin A1c $(\%)$ & -0.040 & 0.772 \\
Changes in fasting blood glucose $(\mathrm{mg} / \mathrm{dL})$ & 0.090 & 0.517 \\
Changes in fasting blood CPR $(\mathrm{ng} / \mathrm{mL})$ & -0.194 & 0.172 \\
Changes in fasting blood glucagon $(\mathrm{pg} / \mathrm{mL})$ & -0.167 & 0.251 \\
Changes in hematocrit $(\%)$ & 0.136 & 0.326 \\
Changes in hemoglobin $(\mathrm{mg} / \mathrm{dL})$ & 0.174 & 0.209 \\
Changes in TG $(\mathrm{mg} / \mathrm{dL})$ & 0.022 & 0.876 \\
Changes in NEFA $(\mu \mathrm{HE} / \mathrm{L})$ & 0.054 & 0.709 \\
Changes in systolic blood pressure $(\mathrm{mmHg})^{*}$ & -0.278 & 0.042 \\
Changes in diastolic blood pressure $(\mathrm{mmHg})$ & -0.165 & 0.232 \\
Changes in HR $($ per minute) & -0.139 & 0.315 \\
Changes in BNP $(\mathrm{pg} / \mathrm{mL})$ & -0.099 & 0.482 \\
Changes in TFA $\left(\mathrm{cm}^{2}\right)^{*}$ & -0.276 & 0.048 \\
Changes in VFA $\left(\mathrm{cm}^{2}\right)$ & -0.115 & 0.416 \\
Changes in SFA $\left(\mathrm{cm}^{2}\right)^{*}$ & -0.333 & 0.016 \\
Changes in total ketone body $(\mu \mathrm{mol} / \mathrm{L})$ & 0.199 & 0.170 \\
Changes in adiponectin $(\mu \mathrm{g} / \mathrm{mL}) *$ & 0.327 & 0.027 \\
\hline
\end{tabular}

${ }^{*} \mathrm{p}<0.05$. LnRHI: natural logarithmic transformation of reactive-hyperemia index, CPR: C-peptide immunoreactivity, TG: triglyceride, NEFA: non-esterified free fatty acid, HR: heart rate, BNP: B-type natriuretic peptide, TFA: total fat area, VFA: visceral fat area, SFA: subcutaneous fat area mass.

SGLT2 inhibitors exerted blood pressure-lowering effects in a previous meta-analysis (26). We also confirmed the beneficial effects of dapagliflozin on the blood pressure in the present study. A previous study showed that 2-day dapagliflozin treatment improved the endothelial function, independent of blood pressure-lowering effects (12). However, we found a significant correlation between the degree of improvement in the endothelial function and the reduction in systolic blood pressure in our six-month study. We speculate that the endothelial function improved by dapagliflozin might subsequently induce the functional recovery of impaired microcirculation and abnormal vasomotion of arterioles with increased vascular tone, thereby leading to a reduction in the systemic blood pressure.

Obesity and fat accumulation are major fundamental pathogenic conditions in patients with T2DM (30). Glucoselowering treatment strategies that can provide a reduction in the body weight and fat mass are thought to have additive clinical advantage in the management of DM (4). The posthoc results of the Look AHEAD study showed the cardiovascular benefits of practically achieved weight reduction during the long-term management of T2DM (31). Matsuzawa et al. reported that the waist circumference, reflecting abdominal obesity, was strongly associated with endothelial dysfunction in patients with metabolic syndrome (32). They also found that the degree of reduction in the waist circumference by lifestyle modifications was substantially correlated with improvement in the endothelial function. The current study showed a significant correlation between the 
dapagliflozin-induced improvement in the endothelial function and changes in the plasma adiponectin levels and abdominal fat mass, particularly SFA, as assessed by abdominal CT. Adiponectin has athero-protective effects and improves the endothelial function (33). Because SGLT2 inhibitors improve the glycemic control via the reduction in the body weight and fat mass (2), SGLT2 inhibitors may be able to achieve cardiovascular benefits by improving the endothelial function, accompanied by a decrease in the abdominal fat mass and an increase in the plasma adiponectin levels. Recent experimental approaches to examining the mechanism underlying the effects of SGLT2 inhibitors on the vascular function have suggested that the effects of SGLT2 inhibitors on the endothelial function may be independent of a reduction in fat $(34,35)$. Further studies are required to clarify the precise mechanisms underlying the effects of SGLT2 inhibitors on the endothelial function.

Adiponectin is secreted by adipocytes, and the decreased plasma levels of adiponectin play an important role in the pathogenesis of T2DM and cardiovascular diseases (33). Adiponectin levels are reported to be inversely correlated with both the total and abdominal fat mass (36). Thiazolidinediones, PPAR $\gamma$ agonists, have been shown to increase the circulating levels of adiponectin in humans (37). We observed increased plasma adiponectin levels in patients receiving dapagliflozin therapy in the present study, although the detailed molecular mechanisms underlying this dapagliflozin-induced elevation remain unclear. A reduction in both the visceral and subcutaneous fat mass helps increase the adiponectin level through lifestyle intervention in patients with T2DM (38). Because adipocytes do not express SGLT2, the direct effects of dapagliflozin on adipocytes may not play a central role in the present results. We instead speculate that the effects of dapagliflozin-induced fat mass reduction via urinary calorie loss may modulate the functions and metabolism of adipocytes, leading to the elevation of plasma adiponectin.

At present, only two clinical studies with dapagliflozin have shown improving effects of SGLT2-inhibitors on the endothelial function $(11,12)$. In in vivo animal models, dapagliflozin treatment (35), empagliflozin treatment (39), and ipragliflozin treatment (34) improved the endothelial function. However, we lack sufficient clinical data at present regarding the SGLT2 inhibitor-induced improvement in the endothelial function as a drug class effect.

Our study has several limitations, including the small number of subjects, non-random allocation, open-label design, relatively short study period, and possible bias in patient selection. More detailed and longer studies are required to validate the effects of SGLT2 inhibitor treatment on improving the endothelial function as demonstrated in the present study. The beneficial effects of SGLT2 inhibitors on the endothelial function should be confirmed in larger, randomized clinical studies (40). This study included patients with uncontrolled T2DM (HbA1c $>7.0 \%)$ as well as patients without severe obesity $\left(\right.$ BMI $\left.>35 \mathrm{~kg} / \mathrm{m}^{2}\right)$. Further studies evaluating the effects of dapagliflozin on the endothelial function should be considered in patients with T2DM with a normal weight, severe obesity, older age, and well-controlled DM. In addition, the detailed mechanisms underlying the dapagliflozin-induced improvement in the endothelial function could not be determined in the present clinical study. We were also unable to measure markers of inflammation and oxidative stress. Prospective studies including these biomarkers may help determine the underlying mechanisms of action of SGLT2 inhibitors.

\section{Conclusion}

Add-on treatment with dapagliflozin significantly improves the glycemic control and endothelial function associated with a reduction in the abdominal fat mass in patients with uncontrolled T2DM.

\section{Author's disclosure of potential Conflicts of Interest (COI).}

Seigo Sugiyama: Honoraria, MSD and AstraZeneca Pharmaceuticals. Hideaki Jinnouchi: Employment/Leadership position/Advisory role, Sanofi U.S., Novo Nordisk, and Eli Lilly Japan; Honoraria, MSD, Astellas Pharma US, Sanofi U.S., Novo Nordisk Pharma, Taishi Toyama Pharmaceutical, Daiichi-Sankyo, Mitsubishi Tanabe Pharma, Eli Lilly Japan, Boehringer Ingelheim Pharmaceuticals, Takeda Pharmaceutical and AstraZeneca Pharmaceuticals.

\section{Acknowledgement}

The authors thank Noriko Matsuda, Mayumi Shimizu, Noriko Nakamura, and Kazue Furuta for their skillful technical assistance.

\section{References}

1. Vallon V. The mechanisms and therapeutic potential of SGLT2 inhibitors in diabetes mellitus. Annu Rev Med 66: 255-270, 2015.

2. Bolinder J, Ljunggren O, Kullberg J, et al. Effects of dapagliflozin on body weight, total fat mass, and regional adipose tissue distribution in patients with type 2 diabetes mellitus with inadequate glycemic control on metformin. J Clin Endocrinol Metab 97: 1020-1031, 2012.

3. Ribola FA, Cancado FB, Schoueri JH, De Toni VF, Medeiros VH, Feder D. Effects of SGLT2 inhibitors on weight loss in patients with type 2 diabetes mellitus. Eur Rev Med Pharmacol Sci 21: 199-211, 2017.

4. Scheen AJ, Van Gaal LF. Combating the dual burden: therapeutic targeting of common pathways in obesity and type 2 diabetes. Lancet Diabetes Endocrinol 2: 911-922, 2014.

5. Tahrani AA, Barnett AH, Bailey CJ. Pharmacology and therapeutic implications of current drugs for type 2 diabetes mellitus. Nat Rev Endocrinol 12: 566-592, 2016.

6. Kaku K, Maegawa H, Tanizawa Y, et al. Dapagliflozin as monotherapy or combination therapy in Japanese patients with type 2 diabetes: an open-label study. Diabetes Ther 5: 415-433, 2014.

7. Zinman B, Wanner C, Lachin JM, et al. Empagliflozin, cardiovascular outcomes, and mortality in type 2 diabetes. $\mathrm{N}$ Engl $\mathrm{J}$ Med 373: 2117-2128, 2015.

8. Neal B, Perkovic V, Mahaffey KW, et al. Canagliflozin and cardiovascular and renal events in type 2 diabetes. $\mathrm{N}$ Engl J Med 377: 644-657, 2017.

9. Heerspink HJ, Perkins BA, Fitchett DH, Husain M, Cherney DZ. Sodium glucose cotransporter 2 inhibitors in the treatment of dia- 
betes mellitus: cardiovascular and kidney effects, potential mechanisms, and clinical applications. Circulation 134: 752-772, 2016.

10. Cherney DZ, Perkins BA, Soleymanlou N, et al. The effect of empagliflozin on arterial stiffness and heart rate variability in subjects with uncomplicated type 1 diabetes mellitus. Cardiovasc Diabetol 13: 28, 2014.

11. Shigiyama F, Kumashiro N, Miyagi M, et al. Effectiveness of dapagliflozin on vascular endothelial function and glycemic control in patients with early-stage type 2 diabetes mellitus: DEFENCE study. Cardiovasc Diabetol 16: 84, 2017.

12. Solini A, Giannini L, Seghieri M, et al. Dapagliflozin acutely improves endothelial dysfunction, reduces aortic stiffness and renal resistive index in type 2 diabetic patients: a pilot study. Cardiovasc Diabetol 16: 138, 2017.

13. Standl E, Schnell O, McGuire DK, Ceriello A, Ryden L. Integration of recent evidence into management of patients with atherosclerotic cardiovascular disease and type 2 diabetes. Lancet Diabetes Endocrinol 5: 391-402, 2017.

14. Matsuzawa Y, Sugiyama S, Sumida H, et al. Peripheral endothelial function and cardiovascular events in high-risk patients. J Am Heart Assoc 2: e000426, 2013.

15. Matsuzawa Y, Kwon TG, Lennon RJ, Lerman LO, Lerman A. Prognostic value of flow-mediated vasodilation in brachial artery and fingertip artery for cardiovascular events: a systematic review and meta-analysis. J Am Heart Assoc 4: e002270, 2015.

16. Tabit CE, Chung WB, Hamburg NM, Vita JA. Endothelial dysfunction in diabetes mellitus: molecular mechanisms and clinical implications. Rev Endocr Metab Disord 11: 61-74, 2010.

17. Matsuzawa Y, Guddeti RR, Kwon TG, Lerman LO, Lerman A. Secondary prevention strategy of cardiovascular disease using endothelial function testing. Circ J 79: 685-694, 2015.

18. Jayawardene D, Ward GM, O'Neal DN, Theverkalam G, MacIsaac AI, MacIsaac RJ. New treatments for type 2 diabetes: cardiovascular protection beyond glucose lowering? Heart Lung Circ 23: 997$1008,2014$.

19. Kurinami N, Sugiyama S, Nishimura $H$, et al. Clinical factors associated with initial decrease in body-fat percentage induced by add-on sodium-glucose co-transporter 2 inhibitors in patient with type 2 diabetes mellitus. Clin Drug Investig 38: 19-27, 2018.

20. Kuvin JT, Patel AR, Sliney KA, et al. Assessment of peripheral vascular endothelial function with finger arterial pulse wave amplitude. Am Heart J 146: 168-174, 2003.

21. Matsubara J, Sugiyama S, Akiyama E, et al. Dipeptidyl peptidase4 inhibitor, sitagliptin, improves endothelial dysfunction in association with its anti-inflammatory effects in patients with coronary artery disease and uncontrolled diabetes. Circ J 77: 1337-1344, 2013.

22. Malavolti M, Mussi C, Poli M, et al. Cross-calibration of eightpolar bioelectrical impedance analysis versus dual-energy X-ray absorptiometry for the assessment of total and appendicular body composition in healthy subjects aged 21-82 years. Ann Hum Biol 30: 380-391, 2003.

23. Kurinami N, Sugiyama S, Yoshida A, et al. Correlation of body muscle/fat ratio with insulin sensitivity using hyperinsulinemiceuglycemic clamp in treatment-naive type 2 diabetes mellitus. Diabetes Res Clin Pract 120: 65-72, 2016.

24. Yoshizumi T, Nakamura T, Yamane M, et al. Abdominal fat: standardized technique for measurement at CT. Radiology 211: 283286, 1999.

25. McCrea CE, Skulas-Ray AC, Chow M, West SG. Test-retest reliability of pulse amplitude tonometry measures of vascular endothelial function: implications for clinical trial design. Vasc Med 17: 29-36, 2012.

26. Mazidi M, Rezaie P, Gao HK, Kengne AP. Effect of sodium- glucose cotransport-2 inhibitors on blood pressure in people with type 2 diabetes mellitus: a systematic review and meta-analysis of 43 randomized control trials with 22528 patients. J Am Heart Assoc 6: 2017.

27. Ray KK, Seshasai SR, Wijesuriya S, et al. Effect of intensive control of glucose on cardiovascular outcomes and death in patients with diabetes mellitus: a meta-analysis of randomised controlled trials. Lancet 373: 1765-1772, 2009.

28. Stojanovic M, Prostran M, Radenkovic M. Thiazolidinediones improve flow-mediated dilation: a meta-analysis of randomized clinical trials. Eur J Clin Pharmacol 72: 385-398, 2016.

29. Torimoto $\mathrm{K}$, Okada $\mathrm{Y}$, Mori $\mathrm{H}$, et al. Effects of exenatide on postprandial vascular endothelial dysfunction in type 2 diabetes mellitus. Cardiovasc Diabetol 14: 25, 2015.

30. Esser N, Legrand-Poels S, Piette J, Scheen AJ, Paquot N. Inflammation as a link between obesity, metabolic syndrome and type 2 diabetes. Diabetes Res Clin Pract 105: 141-150, 2014.

31. Look ARG, Gregg EW, Jakicic JM, et al. Association of the magnitude of weight loss and changes in physical fitness with longterm cardiovascular disease outcomes in overweight or obese people with type 2 diabetes: a post-hoc analysis of the Look AHEAD randomised clinical trial. Lancet Diabetes Endocrinol 4: 913-921, 2016.

32. Matsuzawa Y, Sugiyama S, Sugamura K, et al. Successful diet and exercise therapy as evaluated on self-assessment score significantly improves endothelial function in metabolic syndrome patients. Circ J 77: 2807-2815, 2013.

33. Achari AE, Jain SK. Adiponectin, a therapeutic target for obesity, diabetes, and endothelial dysfunction. Int J Mol Sci 18: E1321, 2017.

34. Salim HM, Fukuda D, Yagi S, Soeki T, Shimabukuro M, Sata M. Glycemic control with ipragliflozin, a novel selective SGLT2 inhibitor, ameliorated endothelial dysfunction in streptozotocininduced diabetic mouse. Front Cardiovasc Med 3: 43, 2016.

35. Gaspari T, Spizzo I, Liu H, et al. Dapagliflozin attenuates human vascular endothelial cell activation and induces vasorelaxation: a potential mechanism for inhibition of atherogenesis. Diab Vasc Dis Res 15: 64-73, 2018.

36. Gavrila A, Chan JL, Yiannakouris N, et al. Serum adiponectin levels are inversely associated with overall and central fat distribution but are not directly regulated by acute fasting or leptin administration in humans: cross-sectional and interventional studies. J Clin Endocrinol Metab 88: 4823-4831, 2003.

37. Yamauchi T, Kamon J, Waki H, et al. The fat-derived hormone adiponectin reverses insulin resistance associated with both lipoatrophy and obesity. Nat Med 7: 941-946, 2001.

38. Zhang C, Luo H, Gao F, Zhang CT, Zhang R. A reduction in both visceral and subcutaneous fats contributes to increased adiponectin by lifestyle intervention in the Diabetes Prevention Program. Acta Diabetol 52: 625-628, 2015.

39. Steven S, Oelze M, Hanf A, et al. The SGLT2 inhibitor empagliflozin improves the primary diabetic complications in ZDF rats. Redox Biol 13: 370-385, 2017.

40. Tanaka A, Shimabukuro M, Okada Y, et al. Rationale and design of a multicenter placebo-controlled double-blind randomized trial to evaluate the effect of empagliflozin on endothelial function: the EMBLEM trial. Cardiovasc Diabetol 16: 48, 2017.

The Internal Medicine is an Open Access article distributed under the Creative Commons Attribution-NonCommercial-NoDerivatives 4.0 International License. To view the details of this license, please visit (https://creativecommons.org/licenses/ by-nc-nd/4.0/).

(C) 2018 The Japanese Society of Internal Medicine Intern Med 57: 2147-2156, 2018 